ALEA, Lat. Am. J. Probab. Math. Stat. 18, 1-15 (2021)

DOI: 10.30757/ALEA.v18-01

\title{
Convergence of the Fleming-Viot process toward the minimal quasi-stationary distribution
}

\author{
Nicolas Champagnat and Denis Villemonais \\ Université de Lorraine, CNRS, Inria, IECL, UMR 7502 \\ F-54000 Nancy, France. \\ E-mail address: Nicolas.Champagnat@inria.fr \\ URL: http://www.iecl. univ-lorraine.fr/ Nicolas. Champagnat/ \\ Université de Lorraine, CNRS, Inria, IECL, UMR 7502 \\ F-54000 Nancy, France. \\ E-mail address: Denis.Villemonais@univ-lorraine.fr \\ URL: http://www.normalesup.org/ villemonais/
}

\begin{abstract}
We prove under mild conditions that the Fleming-Viot process selects the minimal quasi-stationary distribution for Markov processes with soft killing on non-compact state spaces. Our results are applied to multi-dimensional birth and death processes, continuous time Galton-Watson processes and diffusion processes with soft killing.
\end{abstract}

\section{Introduction}

Let $\left(\Omega,\left(\mathcal{F}_{t}\right)_{t \in[0,+\infty},\left(\mathbb{P}_{x}\right)_{x \in E},\left(X_{t}\right)_{t \in[0,+\infty]}\right)$ be a continuous time càdlàg Markov process evolving in a Polish state space $(E, \mathcal{E})$. Let also $\kappa: E \rightarrow \mathbb{R}_{+}$be a bounded measurable function and consider the killed Markov process $\left(Y_{t}\right)_{t \in[0,+\infty)}$ evolving in $E \cup\{\partial\}$, where $\partial \notin E$ is a cemetery point, defined by

$$
Y_{t}= \begin{cases}X_{t} & \text { if } \int_{0}^{t} \kappa\left(X_{s}\right) d s<\zeta \\ \partial & \text { otherwise }\end{cases}
$$

where $\zeta$ is an exponential random variable with parameter 1, independent from $X$. A quasi-stationary distribution for $Y$ is a probability measure $\nu_{Q S D}$ on $E$ such that

$$
\lim _{t \rightarrow+\infty} \mathbb{P}_{\mu}\left(Y_{t} \in \cdot \mid t<\tau_{\partial}\right)=\nu_{Q S D},
$$

for some probability measure $\mu$ on $E$, and where the convergence holds for the total variation distance (for example). The distribution $\nu_{Q S D}$ is called the minimal

Received by the editors December 6th, 2018; accepted July 15th, 2019.

2010 Mathematics Subject Classification. 37A25, 60K35, 60F99.

Key words and phrases. Quasi-stationary distributions, Fleming-Viot processes, birth and death processes, Galton-Watson processes, diffusion processes. 
quasi-stationary distribution of $Y$ if the above convergence holds true for all $\mu=\delta_{x}$, where $x$ runs over $E$ (Asselah and Thai, 2012; Asselah et al., 2016).

This paper is devoted to the study, in a general setting, of the ergodicity and the convergence of a Fleming-Viot type particle system in the long time/large particle limit toward the minimal quasi-stationary distribution of $Y$. In particular, we recover and sensibly improve the results of Asselah et al. (2016) and Villemonais (2015), where adhoc probabilistic and spectral considerations were used to study this problem for continuous time Galton-Watson processes and birth and death processes respectively. The main difficulty in these examples is that the state space is neither compact nor the process comes back from infinity, so that uniqueness of a quasi-stationary distribution may not hold true (see also Asselah and Thai, 2012 for a fine control of the rightmost particle position in a Fleming-Viot type model). In order to control the behaviour of the particle system despite the lack of compactness, we make use of the general results of Champagnat and Villemonais (2017) for the study of quasi-stationary distributions. Our main contributions compared to the previously cited references are the generality of our approach (our framework includes, for instance, the case of multi-dimensional birth and death processes, of multi-type Galton Watson processes and of diffusion processes on $\mathbb{R}^{d}$ ), a new speed of convergence result and a stronger convergence.

Let us describe the dynamics of the Fleming-Viot particle system with $N \geq 2$ particles, which we denote by $\left(X^{1}, X^{2}, \ldots, X^{N}\right)$. The process starts at a position $\left(X_{0}^{1}, X_{0}^{2}, \ldots, X_{0}^{N}\right) \in E^{N}$ and evolves as follows:

- the particles $X^{i}, i=1, \ldots, N$, evolve as independent copies of the process $Y$ until one of them hits $\partial$; this hitting time is denoted by $\tau_{1}$;

- then the (unique) particle hitting $\partial$ at time $\tau_{1}$ jumps instantaneously on the position of a particle chosen uniformly among the $N-1$ remaining ones; this operation is called a rebirth;

- because of this rebirth, the $N$ particles lie in $E$ at time $\tau_{1}$; then the $N$ particles evolve as independent copies of $Y$ and so on.

Note that our settings correspond to the soft killing case, so that, denoting by $\tau_{1}<\tau_{2}<\cdots<\tau_{n}<\cdots$ the sequence of rebirths times and since the rate at which rebirths occur is uniformly bounded from above by $N\|\kappa\|_{\infty}$,

$$
\lim _{n \rightarrow \infty} \tau_{n}=+\infty \text { almost surely. }
$$

Hence, contrarily to the hard killing case (Bieniek et al., 2012), there is no risk for the particle system $\left(X_{t}^{1}, X_{t}^{2}, \ldots, X_{t}^{N}\right)_{t \geq 0}$ to undergo an infinite number of jump in finite time. This guarantees that it is well defined for any time $t \geq 0$ in an incremental way (rebirth after rebirth). We emphasise that, because of the rebirth mechanism, the particle system $\left(X^{1}, X^{2}, \ldots, X^{N}\right)$ evolves in $E^{N}$.

For a fixed number of particles $N \geq 2$ and any function $\varphi$ belonging to the domain of the extended generator $L$ of $X$ (in the sense of Meyn and Tweedie, 1993), one checks that the function $f\left(x_{1}, \ldots, x_{N}\right)=\sum_{i=1}^{N} \varphi\left(x_{i}\right)$ belongs to the domain of the extended generator $L^{N}$ of $\left(X^{1}, \ldots, X^{N}\right)$, with

$$
L^{N} f\left(x_{1}, \ldots, x_{N}\right)=\sum_{i=1}^{N} L \varphi\left(x_{i}\right)+\frac{\kappa\left(x_{i}\right)}{N-1} \sum_{j=1, j \neq i}^{N}\left(\varphi\left(x_{j}\right)-\varphi\left(x_{i}\right)\right) .
$$


This Fleming-Viot type system has been introduced by Burdzy, Holyst, Ingermann and March (Burdzy et al., 1996) and studied in Burdzy et al. (2000), Grigorescu and Kang (2004), Villemonais (2011), Grigorescu and Kang (2012) for multi-dimensional diffusion processes. The study of this system when the underlying Markov process $X$ is a continuous time Markov chain in a countable state space has been initiated in Ferrari and Marić (2007) and followed by Asselah et al. (2011), Asselah et al. (2016), Groisman and Jonckheere (2013b), Asselah and Thai (2012) and Cloez and Thai (2016). We also refer the reader to Groisman and Jonckheere (2013a), where general considerations on the link between the study of such systems and front propagation problems are considered and to Cerou et al. (2016); Delyon et al. (2017) where CLTs for this Fleming-Viot type process have been proved. Note that the Fleming-Viot process considered here is different from the classical Fleming-Viot measure-valued diffusion process (Dawson, 1993), which can be obtained as a limit of particle systems also including mutations and resampling, but with a different parameter scaling. We refer the reader to Ethier and Kurtz (1993) for the long-time behavior of this particular process.

For any $t \geq 0$, we denote by $\mu_{t}^{N}$ the empirical distribution of $\left(X^{1}, X^{2}, \ldots, X^{N}\right)$ at time $t$, defined by

$$
\mu_{t}^{N}=\frac{1}{N} \sum_{i=1}^{N} \delta_{X_{t}^{i}} \in \mathcal{M}_{1}(E)
$$

where $\mathcal{M}_{1}(E)$ is the set of probability measures on $E$. Our aim is to find a tractable condition ensuring: 1) that $Y$ admits a minimal quasi-stationary distribution using the recent general results of Champagnat and Villemonais (2017); 2) that the law of $\mu_{t}^{N}$ converges toward the law of a (random) distribution $\mathcal{X}^{N}$ on $E$ using classical Foster-Lyapunov type criteria (Meyn and Tweedie, 1993); and 3) that this sequence of random distributions converges to the (deterministic) minimal quasi-stationary distribution of $Y$ using the general convergence results of Villemonais (2014).

Our main results are stated in Section 2, illustrated by several examples in Section 3 and proved in Section 4.

\section{Main results}

We first present our main assumptions. Several examples satisfying this requirement are provided in the next section. For the definition of the extended domain of the generator, we refer the reader to Meyn and Tweedie (1993). We recall that a subset $K \in E$ is called a small set for $X$ if there exist a positive time $t_{K}>0$, a positive constant $\alpha_{K}>0$ and a probability measure $\nu_{K}$ on $K$ such that, for all $x \in K$,

$$
\mathbb{P}_{x}\left(X_{t_{K}} \in A\right) \geq \alpha_{K} \nu_{K}(A \cap K), \quad \forall A \subset E .
$$

We also refer the reader to Meyn and Tweedie (1993) and references therein for general considerations on small sets and the related concept of petite sets.

Assumption H. Assume that all compact sets are small sets for $X$. Assume also that there exists a locally bounded function $V: E \rightarrow[1,+\infty)$ in the extended domain of the infinitesimal generator of $X$, with relatively compact level sets and such that

$$
L V(x) \leq-\lambda_{1} V(x)+C, \forall x \in E
$$


for some constant $\lambda_{1}>\|\kappa\|_{\infty}$. Finally, assume that $Y$ satisfies the following property: for all compact set $K \subset E$,

$$
\inf _{t \geq 0} \frac{\inf _{x \in K} \mathbb{P}_{x}\left(Y_{t} \notin \partial\right)}{\sup _{x \in K} \mathbb{P}_{x}\left(Y_{t} \notin \partial\right)}>0
$$

Of course, the two first points of Assumption $\mathrm{H}$ imply that $X$ satisfies a FosterLyapunov type criterion and hence that it is exponentially ergodic. However, this does not guarantee the long-time convergence of the conditional distribution of $Y$. For instance, if $X$ is a birth and death process evolving in $\mathbb{N}=\{1,2,3, \ldots\}$ and if $\kappa(x)=\mathbb{1}_{x=1}$, the exponential ergodicity of $X$ is not sufficient to deduce the long-time convergence of the conditional distribution of $Y$ (as clearly appears in the reference work van Doorn, 1991). The last point of Assumption H allows us to overcome this difficulty (using the recent results of Champagnat and Villemonais, 2017).

Theorem 2.1. If Assumption $H$ holds true, then $Y$ admits a unique quasistationary distribution $\nu_{Q S D}$ such that $\nu_{Q S D}(V)<+\infty$. Moreover, there exist two positive constants $c, \gamma>0$ such that

$$
\left\|\mathbb{P}_{\mu}\left(Y_{t} \in \cdot \mid t<\tau_{\partial}\right)-\nu_{Q S D}\right\|_{T V} \leq c \mu(V) e^{-\gamma t}, \forall t>0,
$$

for any probability distribution $\mu$ on $E$ such that $\mu(V)<\infty$.

Since $V(x)<\infty$ for all $x \in E, \nu_{Q S D}$ is the minimal quasi-stationary distribution of $Y$. Several other properties can be deduced from Assumption $\mathrm{H}$ and Theorem 2.1, as detailed in Champagnat and Villemonais (2017, Section 2).

Remark 2.2. One can weaken Assumption H replacing $\lambda_{1}>\|\kappa\|_{\infty}$ by $\lambda_{1}>$ osc $\kappa$, where osc $\kappa=\sup _{x \in E} \kappa(x)-\inf _{x \in E} \kappa(x)$ is the oscillation of $\kappa$. However, this generalisation does not transfer to the next results.

The next theorem states that the Fleming-Viot process defined in the introduction is exponentially ergodic for $N$ large enough.

Theorem 2.3. Assume that Assumption $H$ is satisfied. Then, for any $N>$ $\frac{\lambda_{1}}{\lambda_{1}-\|\kappa\|_{\infty}}$, the process $\left(X^{1}, \ldots, X^{N}\right)_{t \geq 0}$ is exponentially ergodic, which means that there exists a probability measure $M^{N}$ on $E^{N}$ such that

$$
\left\|\operatorname{Law}\left(X_{t}^{1}, \ldots, X_{t}^{N} \mid\left(X_{0}^{1}, \ldots, X_{0}^{N}\right)=x \in E^{N}\right)-M^{N}\right\|_{T V} \leq C_{N}(x) e^{-\gamma_{N} t},
$$

where $C_{N}: E^{N} \rightarrow[0,+\infty)$ is a non-negative function and $\gamma_{N}>0$ is a constant. Moreover

$$
\int_{E^{N}} \sum_{i=1}^{N} V\left(x_{i}\right) d M^{N}\left(x_{1}, \ldots, x_{N}\right) \leq C /\left(\lambda_{1}-\|\kappa\|_{\infty} N /(N-1)\right) .
$$

Note that, using the above theorem, one deduces that $\mu_{t}^{N}$ also converges in law, when $t \rightarrow+\infty$, to a (random) probability measure $\mathcal{X}^{N}$ on $E$, defined as

$$
\mathcal{X}^{N}=\frac{1}{N} \sum_{i=1}^{N} \delta_{x_{i}}, \text { where } \operatorname{Law}\left(x_{0}, \ldots, x_{N}\right)=M^{N} .
$$


The following last result concludes that, under Assumption $\mathrm{H}$, the sequence of random variables $\left(\mathcal{X}^{N}\right)_{N}$ valued in $\mathcal{M}_{1}(E)$ converges to the minimal quasistationary distribution of $X$. The constant $\gamma$ and the measure $\nu_{Q S D}$ are obtained from Theorem 2.1.

Theorem 2.4. Assume that Assumption $H$ is satisfied. Then there exists a constant $d>0$ such that, for all bounded measurable function $f: E \rightarrow \mathbb{R}$ and all $N>$ $\frac{\lambda_{1}}{\lambda_{1}-\|\kappa\|_{\infty}}$,

$$
\mathbb{E}\left|\mathcal{X}^{N}(f)-\nu_{Q S D}(f)\right| \leq \frac{d}{N^{\alpha}}\|f\|_{\infty},
$$

where $\alpha=\frac{\gamma}{2\left(\|\kappa\|_{\infty}+\gamma\right)}$.

Moreover, for all function $f: E \rightarrow \mathbb{R}$ such that $f(x) / V(x) \rightarrow 0$ when $V(x) \rightarrow$ $+\infty$, we have

$$
\mathbb{E}\left|\mathcal{X}^{N}(f)-\nu_{Q S D}(f)\right| \underset{N \rightarrow+\infty}{\longrightarrow} 0 .
$$

In view of this result, a natural question is to find bounds on a sequence $t_{N} \rightarrow$ $+\infty$ such that $\mu_{t_{N}}^{N}$ approaches $\nu_{Q S D}$ for non-stationary initial conditions. Similar arguments as in inequality (4.5) in the proof of Theorem 2.4 imply that, for all $f: E \rightarrow \mathbb{R}$ such that $\|f\|_{\infty} \leq 1$,

$$
\mathbb{E}_{\left(x_{1}, \ldots, x_{N}\right)}\left|\mu_{t}^{N}(f)-\nu_{Q S D}(f)\right| \leq \frac{C}{\sqrt{N}} e^{\|\kappa\|_{\infty} t}+C e^{-\gamma t} \frac{1}{N} \sum_{i=1}^{N} V\left(x_{i}\right)
$$

for some constant $C$ and all $x_{1}, \ldots, x_{N}$ in $E$. This implies the following

Corollary 2.5. Assume that Assumption $H$ is satisfied and $\sup _{N \geq 2} \mathbb{E}_{M_{0}^{N}}\left[\mu_{0}^{N}(V)\right]<$ $+\infty$ where, for all $N \geq 2, M_{0}^{N}$ is the initial distribution of the $N$-Fleming-Viot system $\left(X^{1}, \ldots, X^{N}\right)$. Then, for all sequence $\left(t_{N}\right)_{N \geq 1}$ such that

$$
t_{N} \underset{N \rightarrow+\infty}{\longrightarrow}+\infty \text { and } \frac{\log N}{2\|\kappa\|_{\infty}}-t_{N} \underset{N \rightarrow+\infty}{\longrightarrow}+\infty,
$$

we have for all bounded measurable $f: E \rightarrow \mathbb{R}$

$$
\mathbb{E}_{M_{0}^{N}}\left|\mu_{t_{N}}^{N}(f)-\nu_{Q S D}(f)\right| \underset{N \rightarrow+\infty}{\longrightarrow} 0 .
$$

\section{Examples}

In this section, we apply our results to multi-dimensional birth and death processes, continuous time Galton-Watson processes, continuous time multi-dimensional Galton-Watson processes and multi-dimensional diffusion processes.

3.1. Multi-dimensional birth and death processes. Let $Y$ be a continuous-time multitype birth and death process, taking values in $E \cup\{\partial\}=\mathbb{N}^{d}$ for some $d \geq 1$, with transition rates

$$
q_{x, y}= \begin{cases}b_{i}(x) & \text { if } y=x+e_{i}, \\ d_{i}(x) & \text { if } y=x-e_{i}, \\ 0 & \text { otherwise }\end{cases}
$$

where $\left(e_{1}, \ldots, e_{d}\right)$ is the canonical basis of $\mathbb{Z}_{+}^{d}$ (where $\left.\mathbb{Z}_{+}=\{0,1, \ldots\}\right)$ ), and $\partial=$ $(0, \ldots, 0)$. We assume that $b_{i}(x)>0$ for all $1 \leq i \leq d$ and all $x \in E$, and $d_{i}(x)>0$ for all $x \in E$ and $1 \leq i \leq d$ such that $x_{i} \geq 1$ (of course, $d_{i}(x)=0$ otherwise). 
The following result provides a general explicit criterion on the parameters of the process ensuring that the results of Section 2 hold true.

Proposition 3.1. If

$$
\frac{1}{|x|} \sum_{i=1}^{d}\left(d_{i}(x)-b_{i}(x)\right) \rightarrow+\infty \quad \text { when }|x| \rightarrow+\infty,
$$

or if there exists $\delta>1$ such that

$$
\sum_{i=1}^{d}\left(d_{i}(x)-\delta b_{i}(x)\right) \rightarrow+\infty \quad \text { when }|x| \rightarrow+\infty,
$$

then Assumption $H$ is satisfied.

Indeed, one can choose $V(x)=|x|=x_{1}+\ldots+x_{d}$ if (3.1) is satisfied, and $V(x)=$ $\exp \left(\varepsilon x_{1}+\cdots+\varepsilon x_{d}\right)$ with $\varepsilon>0$ small enough if (3.2) is satisfied (see Champagnat and Villemonais, 2017, Example 7 for the details) and the fact that the killing rate is bounded by $d_{1}\left(e_{1}\right)+\cdots+d_{d}\left(e_{d}\right)$. The rest of Assumption $\mathrm{H}$ is a simple consequence of the irreducibility of the process and the fact that the state space is discrete. Note that the fact that $V$ belongs to the domain of the extended generator of $X$ can be proved by localization techniques (see for instance the proof of Proposition 2.3 in Champagnat and Villemonais, 2019). This will also be the case for the other applications considered below.

Theorem 2.1 was already obtained in Champagnat and Villemonais (2017), while, as far we know, the convergence of $\left(\mathcal{X}^{N}\right)_{N}$ for multi-dimensional birth and death processes toward $\nu_{Q S D}$ is completely new.

Remark 3.2. The one-dimensional birth and death models considered in Examples 2.7, 2.8 and 2.9 of Villemonais (2015, Section 2) all satisfy Assumption H. These birth and death processes studied in the above mentioned paper evolve in $\mathbb{N}=\{1,2, \ldots\}$, with positive birth rates denoted by $b_{x}, x \in \mathbb{N}$, death rates denoted by $d_{x}, x \geq 2$, and with $\kappa(x)=d_{1} \mathbb{1}_{x=1}>0$. The examples considered are

- $b_{x}=b x^{a}$ and $d_{x}=d x^{a}$ for all $x \geq 1$, where $b<d$ are two positive constants and $a>0$ is fixed,

- $b_{i}=b>0$ and $d_{i}=d>0$ for all $i \geq 2$, where $b<d$ are positive constants, with $d_{1}>0$ such that $(\sqrt{d}-\sqrt{b})^{2}>d_{1}$ and $b_{1}>0$,

- $b_{i}=|\sin (i \pi / 2)| i+1$ and $d_{i}=4 i$ for all $i \geq 1$.

The existence of the Lyapunov function $V$ is already proved in this reference, while the rest of Assumption $\mathrm{H}$ is a simple consequence of the irreducibility of birth and death processes with positive coefficients.

While we do not improve the extent of the domain of attraction of the minimal quasi-stationary distribution, we emphasise that, when Villemonais (2015) relies on the spectral theoretical results derived in van Doorn (1991) and leads to weak convergence of measures, our approach is probabilistic and we prove a stronger exponential convergence toward the quasi-stationary distribution.

Theorem 2.3 and the convergence in law of $\left(\mathcal{X}^{N}\right)_{N}$ toward $\nu_{Q S D}$ (in the weak topology) was already proved in the above reference, but the speed of convergence of Theorem 2.4 is new. 
3.2. Continuous-time Galton Watson processes. A continuous time Galton-Watson process describes the evolution of a population where individuals reproduce independently at rate 1 and whose progeny follows a common law $p$ on $\mathbb{Z}_{+}$(here, the progeny replaces the parent, who dies during the reproduction event). More formally, its dynamic is described by the following infinitesimal generator (with $\partial=0$ the unique absorbing point and $f$ is any bounded function):

$$
L f(x)=\sum_{n \geq-1} q(x, x+n)(f(x+n)-f(x)), \forall x \in \mathbb{Z}_{+}
$$

where the jump rates matrix $q(\cdot, \cdot)$ is given by $q(0, \cdot)=0$ and

$$
q(x, x+n)=x p(n+1) \text { for all } x \geq 1 \text { and } n \geq-1 .
$$

Our first result concerns the existence of a minimal quasi-stationary distribution for this process.

Proposition 3.3. Assume that $p(0)>0$ and $p(\{2,3, \ldots\})>0$, that $p$ admits a first moment $m=\sum_{n=0}^{\infty} n p(n) \in(0,1)$ and that there exists $\alpha>1$ such that $\sum_{n=0}^{\infty} n^{\alpha} p(n)<+\infty$. Then the continuous time Galton-Watson process with reproduction law $p$ admits a unique quasi-stationary distribution $\nu_{Q S D}$ such that $\sum_{x=1}^{\infty} \nu_{Q S D}(\{x\}) x^{\alpha}<+\infty$. Moreover, there exist two positive constants $c, \gamma>0$ such that

$$
\left\|\mathbb{P}_{\mu}\left(Y_{t} \in \cdot \mid t<\tau_{\partial}\right)-\nu_{Q S D}\right\|_{T V} \leq c \mu(V) e^{-\gamma t}, \forall t>0,
$$

for any probability distribution $\mu$ on $E$ such that $\sum_{x=1}^{\infty} \mu(\{x\}) x^{\alpha}<+\infty$.

A similar result was obtained in Asselah et al. (2016) using different methods with the requirement that $\alpha=2$. Our result provides a sharper result by allowing any reproduction law with a moment of order $\alpha>1$. Our result also provides the additional exponential convergence in total variation norm and all the consequences listed in Champagnat and Villemonais (2017).

Before proving the above proposition, we state a criterion implying that the continuous time Galton-Watson process satisfies Assumption H.

Proposition 3.4. Assume that $p(0)>0$ and $p(\{2,3, \ldots\})>0$, that $p$ admits a first moment $m=\sum_{n=0}^{\infty} n p(n) \in(0,1)$ and that there exists $\alpha>p(0) /(1-m)$ such that $\sum_{n=0}^{\infty} n^{\alpha} p(n)<+\infty$. Then Assumption $H$ holds true.

Note that, since $m>1-p(0)$, the assumption on $\alpha$ implies that $\alpha>1$. Thanks to Theorem 1.1 in Asselah et al. (2016), the ergodicity of the Fleming-Viot process and its weak convergence toward the quasi-stationary distribution were already known with the condition that $p$ admits an exponential moment. It is thus improved here by considering reproduction laws admitting a polynomial moment of explicit order and by providing convergence in a stronger sense. One major advantage of our approach is also its flexibility : we will show in the next section how this result easily generalises to continuous time multi-type Galton-Watson processes.

We first prove the second proposition, because its proof is straightforward. Setting $V(x)=x^{\alpha}$, one obtains for all $x \geq 1$,

$$
L V(x)=V(x) \sum_{n=0}^{\infty} x p(n)\left[\left(1+\frac{n-1}{x}\right)^{\alpha}-1\right] \sim_{x \rightarrow+\infty} \alpha(m-1) V(x) .
$$


Since we assumed that $\alpha(m-1)<-p(0)$ and since $p(0)$ is the maximum of the absorption rate in this model, one deduces that the Lyapunov Assumption $\mathrm{H}$ is satisfied. As in the previous example, the rest of Assumption $\mathrm{H}$ is a simple consequence of the irreducibility of the process and the fact that the state space is discrete.

Let us now prove the first proposition. This is a direct consequence of Theorem 5.1 in Champagnat and Villemonais (2017). To apply this result, we only need to prove that the constant

$$
\lambda_{0}:=\inf \left\{\lambda>0, \text { s.t. } \liminf _{t \rightarrow+\infty} e^{\lambda t} \mathbb{P}_{x}\left(Y_{t}=x\right)>0\right\}
$$

is strictly smaller than $\alpha(1-m)$. Note that, because of the irreducibility of the process and the discreteness of the state space, this constant does not depend on $x \in \mathbb{N}$ and we also have for all $L \in \mathbb{N}$

$$
\lambda_{0}=\inf \left\{\lambda>0, \text { s.t. } \exists L>0, \liminf _{t \rightarrow+\infty} e^{\lambda t} \mathbb{P}_{x}\left(Y_{t} \in\{1, \ldots, L\}\right)>0\right\} \text {. }
$$

We first observe that, setting $\varphi(x)=x$ for all $x \in \mathbb{N}$, we have $L \varphi(x)=(m-$ 1) $\varphi(x)$. Dynkin's formula implies that the process $M$ defined by $M_{t}=e^{(1-m) t} \varphi\left(Y_{t}\right)$ is a local martingale. Now, using the fact that, for all $t \geq 0, \mathbb{E}\left(V\left(Y_{s}\right)\right)$ is uniformly bounded over $s \in[0, t]$ (because $L V$ is uniformly bounded from above) and the fact that $\varphi=o(V)$, one deduces that $M$ is uniformly integrable over $[0, t]$. In particular it is a martingale and one deduces that

$$
\mathbb{E}_{x}\left(\varphi\left(Y_{t}\right)\right)=e^{(m-1) t} \varphi(x) \text { for all } x \in \mathbb{N} \text { and all } t \geq 0 .
$$

Now, using the fact that $L V(x) \leq \alpha(m-1) V(x)+C \varphi(x)$ for some positive constant $C>0$, one deduces, using Dynkin's formula that

$$
\begin{aligned}
\mathbb{E}_{x} & \left(e^{(1-m) t} V\left(Y_{t}\right)\right) \\
& \leq V(x)+\int_{0}^{t}\left[(\alpha-1)(m-1) \mathbb{E}_{x}\left(e^{(1-m) s} V\left(Y_{s}\right)\right)+C \mathbb{E}_{x}\left(e^{(1-m) s} \varphi\left(Y_{s}\right)\right)\right] d s \\
& \leq(1+C) V(x)-(\alpha-1)(1-m) \int_{0}^{t} \mathbb{E}_{x}\left(e^{(1-m) s} V\left(Y_{s}\right)\right) d s .
\end{aligned}
$$

Reminding that $\alpha>1$, we deduce that

$$
\mathbb{E}_{x}\left(e^{(1-m) t} V\left(Y_{t}\right)\right) \leq(1+C) V(x), \forall t \geq 0 .
$$

From this inequality and (3.3), one obtains, for all $L \in \mathbb{N}$ and all $t \geq 0$,

$$
\begin{aligned}
\varphi(x) & =e^{(1-m) t} \mathbb{E}_{x}\left(\varphi\left(Y_{t}\right)\right) \\
& \leq e^{(1-m) t} \max _{y \in\{1, \ldots, L\}} \varphi(y) \mathbb{P}_{x}\left(Y_{t} \in\{1, \ldots, L\}\right)+e^{(1-m) t} \mathbb{E}_{x}\left(\frac{V\left(Y_{t}\right)}{L^{\alpha-1}}\right) \\
& \leq e^{(1-m) t} \max _{y \in\{1, \ldots, L\}} \varphi(y) \mathbb{P}_{x}\left(Y_{t} \in\{1, \ldots, L\}\right)+\frac{1+C}{L^{\alpha-1}} V(x) .
\end{aligned}
$$

For a fixed $x \in \mathbb{N}$, one can choose $L$ large enough so that $(1+C) V(x) / L^{\alpha-1} \leq$ $\varphi(x) / 2$ and deduce that

$$
\liminf _{t \rightarrow+\infty} e^{(1-m) t} \mathbb{P}_{x}\left(Y_{t} \in\{1, \ldots, L\}\right)>0,
$$

which implies that $\lambda_{0} \leq(1-m)$ and concludes the proof. 
Remark 3.5. Note that equation (3.3) also immediately implies that $\lambda_{0} \geq(1-m)$ so that we in fact proved that $\lambda_{0}=1-m$. The approach employed here for the study of quasi-stationary distributions is of course a general strategy relying on Champagnat and Villemonais (2017) and on Dynkin's formula that may be useful for the study of several processes. The main point is to dispose of a super harmonic function $\varphi$ and a Lyapunov-type norm-like function $V$ that dominates $\varphi$ at infinity.

3.3. Multi-type continuous time Galton-Watson processes. A multi-type continuous time Galton-Watson process describes the evolution of a population of typed individuals, with $d \geq 1$ possible types denoted by $1, \ldots, d$. These individuals reproduce independently at rate $\lambda_{i}>0$ and their progeny has a law $p_{i}$ on $\mathbb{Z}_{+}^{d}$ (here again, the progeny replaces the parent, who dies during the reproduction event), depending on the type $i \in\{1, \ldots, d\}$ of the parent. Its dynamic is thus described by the following infinitesimal generator (where $f$ is any bounded function):

$$
L f(x)=\sum_{i=1}^{d} \sum_{n \in \mathbb{Z}_{+}^{d}} q_{i}\left(x, x+n-e_{i}\right)\left(f\left(x+n-e_{i}\right)-f(x)\right), \quad \forall x \in \mathbb{Z}_{+}^{d}
$$

where $\left(e_{1}, \ldots, e_{d}\right)$ is the canonical basis of $\mathbb{Z}_{+}^{d}$ and the jump rates matrix $q_{i}(\cdot, \cdot)$ is given by $q_{i}(x, \cdot)=0$ if $x_{i}=0$ and

$$
q_{i}\left(x, x+n-e_{i}\right)=\lambda_{i} x_{i} p_{i}(n) \text { otherwise. }
$$

We also assume that $p_{i}$ is such that the associated Galton-Watson process is irreducible in $\mathbb{Z}_{+}^{d} \backslash\{0\}$, so that $\partial=(0, \ldots, 0)$ is the unique absorbing subset of $\mathbb{Z}_{+}^{d}$.

In the following proposition, given such a rate $\lambda_{i}$ and a law $p_{i}$ on $\mathbb{Z}_{+}^{d}$, we denote by $M$ the matrix of mean offsprings defined y $M_{i j}=\lambda_{i} \sum_{n \in \mathbb{Z}_{+}^{d}} n_{j} p_{i}(n)$ and by $Q$ the matrix defined by $Q_{i j}=M_{i j}-\delta_{i j} \lambda_{i}$. Note that the irreducibility of the Galton-Watson process implies the irreducibility and aperiodicity of $e^{t Q}$ and hence, by Perron-Frobenius theorem, the existence of a positive right-eigenvector $v \in(0,+\infty)^{d}$ associated to the spectral radius $\rho \in \mathbb{R}$. Recall that $\rho<0$ corresponds to the subcritical case.

Proposition 3.6. Assume that $\left(\lambda_{i}\right)_{i \in\{1, \ldots, d\}}$ and $\left(p_{i}\right)_{i \in\{1, \ldots, d\}}$ are such that the associated multi-type continuous time Galton-Watson process is irreducible in $\mathbb{Z}_{+}^{d} \backslash$ $\{0\}$. Assume also that the spectral radius $\rho$ of the matrix $Q$ is negative and that $p_{i}$ admits a moment of order $\alpha>\max _{i} p_{i}(0) /(-\rho)$, then Assumption $H$ is satisfied by the multi-type continuous time Galton-Watson process with reproduction laws $\left(p_{i}\right)_{i \in\{1, \ldots, d\}}$ and reproduction rates $\left(\lambda_{i}\right)_{i \in\{1, \ldots, d\}}$.

Since the state space is discrete and the process is assumed to be irreducible, the only difficulty is to prove the existence of a function $V$ such that $L V(x) \leq$ $-\lambda_{1} V(x)+C$ for some constants $\lambda_{1}>\max _{i} p_{i}(0)$ (note that $\max _{i} p_{i}(0)$ is the maximal rate of absorption for our process). In order to do so, one simply observes 
that, setting $V(x)=\left(\sum_{i=1}^{d} v_{i} x_{i}\right)^{\alpha}$ for all $x \in \mathbb{Z}_{+}^{d}$,

$$
\begin{aligned}
L V(x) & =V(x) \sum_{i=1}^{d} \sum_{n \in \mathbb{Z}_{+}^{d}} x_{i} \lambda_{i} p_{i}(n)\left[\left(1+\frac{\sum_{j=1}^{d} v_{j}\left(n_{j}-\delta_{i j}\right)}{\sum_{j=1}^{d} v_{j} x_{j}}\right)^{\alpha}-1\right] \\
& \sim_{|x| \rightarrow+\infty} \frac{\alpha V(x)}{\sum_{j=1}^{d} v_{j} x_{j}} \sum_{i=1}^{d} x_{i} \sum_{j=1}^{d} \sum_{n \in \mathbb{Z}_{+}^{d}} p_{i}(n) v_{j} \lambda_{i}\left(n_{j}-\delta_{i j}\right) \\
& =\frac{\alpha V(x)}{\sum_{j=1}^{d} v_{j} x_{j}} \sum_{i=1}^{d} x_{i} \sum_{j=1}^{d} q_{i j} v_{j}=\alpha \rho V(x),
\end{aligned}
$$

so that $V$ satisfies the Lyapunov-type inequality of Assumption $\mathrm{H}$.

3.4. Diffusion processes with soft killing. In this section, we consider the case of a multi-dimensional diffusion process with soft killing and provide a sufficient criterion for Assumption $\mathrm{H}$ to apply. Let $\left(X_{t}\right)_{t \in[0,+\infty)}$ be the solution in $E=\mathbb{R}^{d}$ to the stochastic differential equation

$$
d X_{t}=\sigma\left(X_{t}\right) d B_{t}+b\left(X_{t}\right) d t
$$

where $B$ is a standard $r$-dimensional Brownian motion, $\sigma: \mathbb{R}^{d} \rightarrow \mathbb{R}^{d \times r}$ and $b: \mathbb{R}^{d} \mapsto$ $\mathbb{R}^{d}$ are locally Hölder-continuous and $a=\sigma \sigma^{*}$ is bounded and locally uniformly elliptic. The process $Y$ is subject to an additional soft killing $\kappa: \mathbb{R}^{d} \mapsto[0,+\infty)$, which is assumed to be uniformly bounded.

Proposition 3.7. If there exist $\beta>0$ and $\gamma>0$ such that

$$
\limsup _{|x| \rightarrow+\infty} \frac{\langle b(x), x\rangle}{\langle x, x\rangle^{1 / 2}} \leq-\beta \quad \text { and } \quad \sum_{i j} a_{i j}(x) x_{i} x_{j} \leq \gamma\langle x, x\rangle,
$$

and such that $\beta^{2}>2 \gamma\|\kappa\|_{\infty}$, then Assumption $H$ is satisfied with $V: x \in \mathbb{R}^{d} \mapsto$ $\exp \left(\rho\langle x, x\rangle^{1 / 2}\right)$, where $\rho>0$ is such that $\rho^{2} \gamma / 2+\|\kappa\|_{\infty}<\beta \rho$.

The fact that Assumption $\mathrm{H}$ is satisfied is in fact a consequence of the material included in Section 4.2, Example 3 of Champagnat and Villemonais (2017): the property on small sets and absorption probabilities are both consequences of the Harnack inequalities proved therein (where they are used to prove the similar properties (F1) and (F3)). The computation of $L V$ is only provided in a simpler case, so we give details below: fix $\varepsilon>0$ such that $\rho^{2} \gamma / 2+\|\kappa\|_{\infty}<(\beta-2 \varepsilon) \rho$. Then, for $|x|$ large enough,

$$
\begin{aligned}
& L V(x) \\
& =\sum_{i=1}^{d} \rho V(x) \frac{\langle b(x), x\rangle}{\langle x, x\rangle^{1 / 2}}+\frac{1}{2} \sum_{1 \leq i, j \leq d} a_{i j}(x) V(x)\left[\rho^{2} \frac{x_{i} x_{j}}{\langle x, x\rangle}-\rho \frac{x_{i} x_{j}}{\langle x, x\rangle^{3 / 2}}+\rho \frac{\delta_{i j}}{\langle x, x\rangle^{1 / 2}}\right] \\
& \leq V(x)\left((-\beta+\varepsilon) \rho+\frac{\rho^{2} \gamma}{2}+\frac{d \rho\|a\|_{\infty}}{\langle x, x\rangle^{1 / 2}}\right) .
\end{aligned}
$$

Therefore, $L V(x) \leq-\left(\|\kappa\|_{\infty}+\varepsilon \rho\right) V(x)$ for $|x|$ large enough, so $V$ satisfies the Lyapunov-type part of Assumption H. 


\section{Proofs}

4.1. Proof of Theorem 2.1. Step 1. Quasi-stationary behaviour of $\left(Y_{n}\right)_{n \in \mathbb{N}}$. Let us first prove that the skeleton $\left(Y_{n}\right)_{n \in \mathbb{N}}$ admits a minimal quasi-stationary distribution, by proving that Assumption E in Champagnat and Villemonais (2017) is satisfied and hence that Theorem 2.1 therein applies. Fix $\lambda \in\left(\lambda_{1},\|\kappa\|_{\infty}\right)$. One deduces from Assumption H, Dynkin's formula, classical upper bound techniques (see for instance Lemma 7 in Mailler and Villemonais (2018)) that, for all $x \in E$ and all $t \geq 0$,

$$
\mathbb{E}_{x}\left(V\left(Y_{t}\right) \mathbb{1}_{t<\tau_{\partial}}\right) \leq \mathbb{E}_{x}\left(V\left(X_{t}\right)\right) \leq \frac{C}{\lambda_{1}-\lambda} \vee\left(e^{-\lambda t} V(x)\right) .
$$

We define the set $K:=\left\{x \in E, V(x) \leq\left(2 \vee e^{\lambda}\right) C /\left(\lambda_{1}-\lambda\right)\right\}$, which is relatively compact by Assumption H. Our aim is to prove Assumption E in Champagnat and Villemonais (2017) with this set $K, \varphi_{1}=V, \varphi_{2}=\mathbb{1}_{E}, \theta_{1}=e^{-\lambda}$ and $\theta_{2}=e^{-\|\kappa\|_{\infty}}$.

Let us first check that Assumption (E1) holds for any $n_{1} \geq t_{K}+\frac{\ln 2}{\lambda}$ (here $t_{K}>0$ is the positive time appearing in the small set property for $K$ ). Note that Assumption (E1) is actually simply the small set property for $K$, but we will need this stronger version in the sequel. We deduce from the definition of $K$ and from the above inequality that, for all $x \in K$,

$$
\mathbb{P}_{x}\left(X_{n_{1}-t_{K}} \notin K\right) \leq \frac{\mathbb{E}_{x}\left(V\left(X_{n_{1}-t_{K}}\right)\right)}{2 C /\left(\lambda_{1}-\lambda\right)} \leq 1 / 2,
$$

In particular, using Markov's property at time $n_{1}-t_{K}$ and the fact that $K$ is a small set for $X$, we obtain

$$
\mathbb{P}_{x}\left(X_{n_{1}} \in A\right) \geq \frac{\alpha_{K}}{2} \nu_{K}(A \cap K),
$$

and hence that

$$
\mathbb{P}_{x}\left(Y_{n_{1}} \in A\right) \geq \frac{\alpha_{K} e^{-\|\kappa\|_{\infty} n_{1}}}{2} \nu_{K}(A \cap K)
$$

for all $n_{1} \geq t_{K}+\frac{\ln 2}{\lambda}$.

We now prove that (E2) is satisfied. Using (4.1), we obtain, for all $x \in E$,

$$
\mathbb{E}_{x}\left(V\left(Y_{1}\right) \mathbb{1}_{1<\tau_{\partial}}\right) \leq \theta_{1} V(x)+\frac{2 C}{\lambda_{1}-\lambda} \mathbb{1}_{K}(x) .
$$

Also, we have

$$
\mathbb{E}_{x}\left(\mathbb{1}_{Y_{1} \in E} \mathbb{1}_{1<\tau_{\partial}}\right) \geq e^{-\|\kappa\|_{\infty}} \mathbb{E}_{x}\left(\mathbb{1}_{X_{1} \in E}\right)=e^{-\|\kappa\|_{\infty}} .
$$

Hence, since we set $\varphi_{1}=V, \varphi_{2}=\mathbb{1}_{E}, \theta_{1}=e^{-\lambda}$ and $\theta_{2}=e^{-\|\kappa\|_{\infty}}$, one deduces that Assumption (E2) is satisfied.

Since $K$ is a relatively compact set, Assumption (E3) is an immediate consequence of the last part of Assumption $\mathrm{H}$.

Finally, setting $n_{4}(x)=\left\lceil t_{K}+\frac{\ln 2}{\lambda}\right\rceil$ for all $x \in K$, one deduces from (4.2) that Assumption (E4) holds true.

We deduce from Champagnat and Villemonais (2017, Theorem 2.1) that there exist a quasi-stationary distribution $\nu_{Q S D}$ for $\left(Y_{n}\right)_{n \in \mathbb{N}}$ and two constants $c^{\prime}>0$ and $\gamma>0$ such that, for all probability measure $\mu$ on $E$ and all $n \geq 0$,

$$
\left\|\mathbb{P}_{\mu}\left(Y_{n} \in \cdot \mid n<\tau_{\partial}\right)-\nu_{Q S D}\right\|_{T V} \leq c^{\prime} \mu(V) e^{-\gamma n} .
$$


Step 2. Conclusion. Our aim is now to prove that $\left(Y_{t}\right)_{t>0}$ exhibits the same quasi-stationary behaviour as $\left(Y_{n}\right)_{n \in \mathbb{N}}$. Let $\mu$ be probability measure on $E$ such that $\mu(V)<\infty$, fix $t \geq 0$ and set $s=t-\lfloor t\rfloor \in[0,1)$. The law $\mu_{s}$ of $Y_{s}$ satisfies (we use the fact that the killing rate of $Y$ is bounded by $\|\kappa\|_{\infty}$, the fact that $L V \leq C$ and Dynkin's formula)

$$
\mu_{s}(V)=\mathbb{E}_{\mu}\left(V\left(Y_{s}\right) \mid s<\tau_{\partial}\right) \leq e^{\|\kappa\|_{\infty} s} \mathbb{E}_{\mu}\left(V\left(X_{s}\right)\right) \leq e^{\|\kappa\|_{\infty}+C} \mu(V) .
$$

From (4.3), we deduce that

$$
\left\|\mathbb{P}_{\mu_{s}}\left(Y_{\lfloor t\rfloor} \in \cdot \mid\lfloor t\rfloor<\tau_{\partial}\right)-\nu_{Q S D}\right\|_{T V} \leq c^{\prime} e^{\|\kappa\|_{\infty}+C} \mu(V) e^{-\gamma\lfloor t\rfloor} .
$$

Setting $c=c^{\prime} e^{\|\kappa\|_{\infty}+C+\gamma}$, one deduces from Markov property at time $s$ and from the above inequality that, for all $t \geq 0$,

$$
\left\|\mathbb{P}_{\mu}\left(Y_{t} \in \cdot \mid t<\tau_{\partial}\right)-\nu_{Q S D}\right\|_{T V} \leq c \mu(V) e^{-\gamma t}
$$

This concludes the proof of Theorem 2.1.

Remark 4.1. One could have been tempted to use Theorem 3.5 in Champagnat and Villemonais (2017) which originally deals with continuous time Markov processes and allows bounded functions for the equivalent of $V$. However, this latitude comes with a far greater complexity and, in particular, one needs to check that the strong Markov property is satisfied at the entry time of $K$ (or, in the present case, a superset of $K$ which is also a small set), which can be quite challenging to prove in general (especially for discontinuous processes).

Since we only consider norm-like functions $V$ in this paper, we can drop this technical requirement, which simplifies the verification of our assumptions. Note that the proofs of Theorems 2.3 and 2.4 also make use of the fact that $V$ is a norm-like function.

4.2. Proof of Theorem 2.3. We define the function $f: E^{N} \rightarrow \mathbb{R}$ by $f(x)=$ $\sum_{i=1}^{N} V\left(x_{i}\right)$. We have, for all $x=\left(x_{1}, \ldots, x_{N}\right) \in E^{N}$,

$$
L^{N} f(x)=\sum_{i=1}^{N} L V\left(x_{i}\right)+\sum_{i=1}^{N} \kappa\left(x_{i}\right) \frac{1}{N-1} \sum_{j=1, j \neq i}^{N} V\left(x_{j}\right)-V\left(x_{i}\right) .
$$

Assumption $\mathrm{H}$ implies that

$$
L^{N} f(x) \leq-\left(\lambda_{1}-\frac{N}{N-1}\|\kappa\|_{\infty}\right) f(x)+C,
$$

where $\lambda_{1}-\frac{N}{N-1}\|\kappa\|_{\infty}$ is positive since $N>\frac{\lambda_{1}}{\lambda_{1}-\|\kappa\|_{\infty}}$ by assumption.

For any constant $k>0$, the set of $N$-tuples $x \in E^{N}$ such that $f(x) \leq k$ is a small set for the Fleming-Viot process, because the level sets of $V$ are small sets for $X$ and because of the boundedness of $\kappa$. Thus, using the Foster Lyapunov criterion of Meyn and Tweedie (1993, Theorem 6.1, p.536) (see also Hairer, 2010, Proposition 1.4 for a simplified account on the subject), we deduce that the Fleming-Viot process is exponentially ergodic and, denoting by $M^{N}$ its unique stationary distribution, we also have

$$
\int_{E^{N}} f(x) d M^{N}(x) \leq C /\left(\lambda_{1}-\frac{N}{N-1}\|\kappa\|_{\infty}\right) .
$$

This concludes the proof of Theorem 2.3. 
4.3. Proof of Theorem 2.4. Since the rebirth rate $\kappa$ is uniformly bounded, it is well known (see for instance Villemonais, 2014; Cloez and Thai, 2016; Cerou et al., 2016) that there exists a constant $d_{0}>0$ such that, for all $N \geq 2$ and all bounded measurable functions $f: E \rightarrow \mathbb{R}$,

$$
\mathbb{E}\left|\mu_{t}^{N}(f)-\mathbb{E}_{\mu_{0}^{N}}\left(f\left(Y_{t}\right) \mid t<\tau_{\partial}\right)\right| \leq \frac{d_{0}}{\sqrt{N}}\|f\|_{\infty} e^{\|\kappa\|_{\infty} t}
$$

In particular, one deduces that, for all $t \geq 0$ (we use the stationarity of $M^{N}$ for the first and second equality and the results of Theorem 2.1 for the second inequality),

$$
\begin{aligned}
\mathbb{E}\left(\left|\mathcal{X}^{N}(f)-\nu_{Q S D}(f)\right|\right)= & \mathbb{E}_{M^{N}}\left|\mu_{t}^{N}(f)-\nu_{Q S D}(f)\right| \\
\leq & \mathbb{E}_{M^{N}}\left|\mu_{t}^{N}(f)-\mathbb{E}_{\mu_{0}^{N}}\left(f\left(Y_{t}\right) \mid t<\tau_{\partial}\right)\right| \\
& \quad+\mathbb{E}_{M^{N}}\left|\mathbb{E}_{\mu_{0}^{N}}\left(f\left(Y_{t}\right) \mid t<\tau_{\partial}\right)-\nu_{Q S D}(f)\right| \\
= & \mathbb{E}_{M^{N}}\left|\mu_{t}^{N}(f)-\mathbb{E}_{\mu_{0}^{N}}\left(f\left(Y_{t}\right) \mid t<\tau_{\partial}\right)\right| \\
& \quad+\mathbb{E}\left|\mathbb{E}_{\mathcal{X}^{N}}\left(f\left(Y_{t}\right) \mid t<\tau_{\partial}\right)-\nu_{Q S D}(f)\right| \\
\leq & \frac{d_{0}}{\sqrt{N}}\|f\|_{\infty} e^{\|\kappa\|_{\infty} t}+c\|f\|_{\infty} e^{-\gamma t} \mathbb{E}\left(\mathcal{X}^{N}(V)\right) \\
\leq & \frac{d_{0}}{\sqrt{N}}\|f\|_{\infty} e^{\|\kappa\|_{\infty} t}+C c\|f\|_{\infty} \frac{e^{-\gamma t}}{\lambda_{1}-\|\kappa\|_{\infty} N /(N-1)},
\end{aligned}
$$

where the last inequality follows from Theorem 2.3. In particular, choosing $t=$ $\frac{\ln N}{2\left(\|\kappa\|_{\infty}+\gamma\right)}$, one deduces that there exists a constant $d>0$ such that

$$
\mathbb{E}\left(\left|\mathcal{X}^{N}(f)-\nu_{Q S D}(f)\right|\right) \leq d\|f\|_{\infty} / N^{\alpha},
$$

where $\alpha=\frac{\gamma}{2\left(\|\kappa\|_{\infty}+\gamma\right)}$.

In order to conclude the proof of Theorem 2.4, fix $f: E \rightarrow \mathbb{R}_{+}$such that $f=o(V)$. Then, since $\mathbb{E}\left(\mathcal{X}^{N}(V)\right)$ is uniformly bounded, one deduces that $f$ is uniformly integrable with respect to this sequence of measures : for all $\delta>0$,

$$
\begin{aligned}
\mathbb{E}\left(\left|\mathcal{X}^{N}(f)-\nu_{Q S D}(f)\right|\right) \leq \mathbb{E}\left(\mid \mathcal{X}^{N}(\right. & \left.\left.f \mathbb{1}_{V \leq \delta}\right)-\nu_{Q S D}\left(f \mathbb{1}_{V \leq \delta}\right) \mid\right) \\
& +\mathbb{E}\left(\left|\mathcal{X}^{N}\left(f \mathbb{1}_{V>\delta}\right)\right|\right)+\mathbb{E}\left(\nu_{Q S D}\left(V \mathbb{1}_{V \leq \delta}\right)\right) .
\end{aligned}
$$

The first term in the right hand side goes to zero when $N \rightarrow+\infty$ (because of the above analysis), while the second and third terms converge to 0 when $\delta \rightarrow+\infty$, uniformly in $N$. This implies that the left hand side converges to 0 when $N \rightarrow+\infty$.

This concludes the proof of Theorem 2.4.

\section{References}

A. Asselah, P. A. Ferrari and P. Groisman. Quasistationary distributions and Fleming-Viot processes in finite spaces. J. Appl. Probab. 48 (2), 322-332 (2011). MR2840302.

A. Asselah, P. A. Ferrari, P. Groisman and M. Jonckheere. Fleming-Viot selects the minimal quasi-stationary distribution: the Galton-Watson case. Ann. Inst. Henri Poincaré Probab. Stat. 52 (2), 647-668 (2016). MR3498004.

A. Asselah and M.-N. Thai. A note on the rightmost particle in a Fleming-Viot process. ArXiv Mathematics e-prints (2012). arXiv: 1212.4168. 
M. Bieniek, K. Burdzy and S. Finch. Non-extinction of a Fleming-Viot particle model. Probab. Theory Related Fields 153 (1-2), 293-332 (2012). MR2925576.

K. Burdzy, R. Hołyst, D. Ingerman and P. March. Configurational transition in a Fleming-Viot-type model and probabilistic interpretation of Laplacian eigenfunctions. J. Phys. A 29 (11), 2633-2642 (1996). DOI: 10.1088/0305-4470/29/11/004.

K. Burdzy, R. Hołyst and P. March. A Fleming-Viot particle representation of the Dirichlet Laplacian. Comm. Math. Phys. 214 (3), 679-703 (2000). MR1800866.

F. Cerou, B. Delyon, A. Guyader and M. Rousset. A central limit theorem for fleming-viot particle systems with soft killing. ArXiv Mathematics e-prints (2016). arXiv: 1611.00515.

N. Champagnat and D. Villemonais. General criteria for the study of quasistationarity. ArXiv Mathematics e-prints (2017). arXiv: 1712.08092.

N. Champagnat and D. Villemonais. Lyapunov criteria for uniform convergence of conditional distributions of absorbed Markov processes. ArXiv Mathematics e-prints (2019). arXiv: 1704.01928v2.

B. Cloez and M.-N. Thai. Quantitative results for the Fleming-Viot particle system and quasi-stationary distributions in discrete space. Stochastic Process. Appl. 126 (3), 680-702 (2016). MR3452809.

D. A. Dawson. Measure-valued Markov processes. In École d'Été de Probabilités de Saint-Flour XXI-1991, volume 1541 of Lecture Notes in Math., pages 1-260. Springer, Berlin (1993). MR1242575.

B. Delyon, F. Cérou, A. Guyader and M. Rousset. A Central Limit Theorem for Fleming-Viot Particle Systems with Hard Killing. ArXiv Mathematics e-prints (2017). arXiv: 1709.06771.

E. A. van Doorn. Quasi-stationary distributions and convergence to quasistationarity of birth-death processes. Adv. in Appl. Probab. 23 (4), 683-700 (1991). MR1133722.

S. N. Ethier and T. G. Kurtz. Fleming-Viot processes in population genetics. SIAM J. Control Optim. 31 (2), 345-386 (1993). MR1205982.

P. A. Ferrari and N. Marić. Quasi stationary distributions and Fleming-Viot processes in countable spaces. Electron. J. Probab. 12, no. 24, 684-702 (2007). MR2318407.

I. Grigorescu and M. Kang. Hydrodynamic limit for a Fleming-Viot type system. Stochastic Process. Appl. 110 (1), 111-143 (2004). MR2052139.

I. Grigorescu and M. Kang. Immortal particle for a catalytic branching process. Probab. Theory Related Fields 153 (1-2), 333-361 (2012). MR2925577.

P. Groisman and M. Jonckheere. Front propagation and quasi-stationary distributions: the same selection principle? ArXiv Mathematics e-prints (2013a). arXiv: 1304.4847.

P. Groisman and M. Jonckheere. Simulation of quasi-stationary distributions on countable spaces. Markov Process. Related Fields 19 (3), 521-542 (2013b). MR3156964.

M. Hairer. Convergence of Markov Processes (2010). Lecture notes, available on-line at www.hairer.org/notes/Convergence.pdf.

C. Mailler and D. Villemonais. Stochastic approximation on non-compact measure spaces and application to measure-valued Pólya processes. ArXiv Mathematics e-prints (2018). arXiv: 1809.01461. 
S. P. Meyn and R. L. Tweedie. Stability of Markovian processes. III. FosterLyapunov criteria for continuous-time processes. Adv. in Appl. Probab. 25 (3), 518-548 (1993). MR1234295.

D. Villemonais. Interacting particle systems and Yaglom limit approximation of diffusions with unbounded drift. Electron. J. Probab. 16, no. 61, 1663-1692 (2011). MR2835250.

D. Villemonais. General approximation method for the distribution of Markov processes conditioned not to be killed. ESAIM Probab. Stat. 18, 441-467 (2014). MR3333998.

D. Villemonais. Minimal quasi-stationary distribution approximation for a birth and death process. Electron. J. Probab. 20, no. 30, 18 (2015). MR3325100. 